University of Wollongong

Research Online

Faculty of Engineering and Information

Faculty of Engineering and Information

Sciences - Papers: Part B

Sciences

2019

Characterisation and evaluation of a new phase change enhanced working solution for liquid desiccant cooling systems

Haoshan Ren

University of Wollongong, hr681@uowmail.edu.au

Zhenjun $\mathrm{Ma}$

University of Wollongong, zhenjun@uow.edu.au

Stefan Gschwander

Fraunhofer Institute for Solar Energy Systems ISE

Follow this and additional works at: https://ro.uow.edu.au/eispapers1

Part of the Engineering Commons, and the Science and Technology Studies Commons

Research Online is the open access institutional repository for the University of Wollongong. For further information contact the UOW Library: research-pubs@uow.edu.au 


\title{
Characterisation and evaluation of a new phase change enhanced working solution for liquid desiccant cooling systems
}

\author{
Abstract \\ Desiccant solutions play an essential role in desiccant cooling systems to absorb moisture from the \\ process air. This paper presents the characterisation of a new working solution for liquid desiccant \\ cooling systems. The new working solution was prepared through dispersion of micro-encapsulated \\ phase change materials (MPCMs) into lithium chloride ( $\mathrm{LiCl}$ ) desiccant solutions to ensure that the \\ dehumidification process was achieved under a low temperature condition and to improve thermal \\ capacity and moisture removal efficiency of the mixture. The properties of the new solution, including \\ density, enthalpy-temperature relationship, particle size distribution, thermal conductivity, and vapour \\ pressure were characterised through either experimental tests or theoretical analysis. It was shown that \\ the density and thermal conductivity of the new working solution slightly decreased with the increase of \\ the mass fraction of the MPCMs in the mixture. The thermal capacity of the new working solution \\ substantially increased in the melting temperature range of the MPCMs used. The vapour pressure of the \\ new working solution decreased due to the existence of the MPCM particles. It is expected that the \\ dehumidification efficiency of adiabatic dehumidifiers can be potentially improved when using this new \\ working solution due to the decreased vapour pressure and increased thermal capacity of the phase \\ change enhanced desiccant solution. \\ Disciplines \\ Engineering | Science and Technology Studies

\section{Publication Details} \\ Ren, H., Ma, Z. \& Gschwander, S. (2019). Characterisation and evaluation of a new phase change \\ enhanced working solution for liquid desiccant cooling systems. Applied Thermal Engineering, 150 \\ 1197-1205.
}


1 Characterisation and evaluation of a new phase change enhanced working solution for liquid desiccant cooling systems

Haoshan Ren ${ }^{1}$, Zhenjun $\mathrm{Ma}^{1,{ }^{*}}$, Stefan Gschwander ${ }^{2}$

${ }^{1}$ Sustainable Buildings Research Centre, University of Wollongong, 2522, Australia

${ }^{2}$ Fraunhofer Institute for Solar Energy Systems, Freiburg, 79110, Germany

*Email: zhenjun@uow.edu.au

.

Abstract: Desiccant solutions play an essential role in desiccant cooling systems to absorb moisture from the process air. This paper presents the characterisation of a new working solution for liquid desiccant cooling systems. The new working solution was prepared through dispersion of micro-encapsulated phase change materials (MPCMs) into lithium chloride ( $\mathrm{LiCl})$ desiccant solutions to ensure that the dehumidification process was achieved under a low temperature condition and to improve thermal capacity and moisture removal efficiency of the mixture. The properties of the new solution, including density, enthalpy-temperature relationship, particle size distribution, thermal conductivity, and vapour pressure were characterised through either experimental tests or theoretical analysis. It was shown that the density and thermal conductivity of the new working solution slightly decreased with the increase of the mass fraction of the MPCMs in the mixture. The thermal capacity of the new working solution substantially increased in the melting temperature range of the MPCMs used. The vapour pressure of the new working solution decreased due to the existence of the MPCM particles. It is expected that the dehumidification efficiency of adiabatic dehumidifiers can be 
22 potentially improved when using this new working solution due to the decreased vapour

23 pressure and increased thermal capacity of the PCM-LiCl desiccant solution.

24 Keywords: Desiccant cooling; phase change material; new working solution; characterisation;

25 vapour pressure.

26

27 Nomenclature

$28 d \quad$ diameter $(\mathrm{m})$

$29 \quad h \quad$ enthalpy $(\mathrm{J} / \mathrm{kg})$

$30 \quad k \quad$ thermal conductivity $(\mathrm{W} / \mathrm{m} \mathrm{K})$

$31 \quad m \quad$ mass $(\mathrm{kg})$

$32 \quad P \quad$ vapour pressure $(\mathrm{Pa})$

$33 t \quad$ time $(\mathrm{s})$

$34 \quad T \quad$ temperature $\left({ }^{\circ} \mathrm{C}\right)$

$35 x_{m} \quad$ mass fraction

$36 x_{v} \quad$ volume fraction

37

38 Greek symbols

$39 \alpha \quad$ coefficient

$40 \quad \alpha_{R} \quad$ equivalent thermal conductivity depression

$41 \quad \beta \quad$ coefficient

$42 \theta \quad$ reduced temperature 
$43 \pi \quad$ coefficient

$44 \quad \rho \quad$ density $\left(\mathrm{kg} / \mathrm{m}^{3}\right)$

45

46 Subscripts

$47 \quad a \quad$ air

$48 \quad c \quad$ core

$49 \quad$ equivalent

$50 \mathrm{LiCl} \quad$ lithium chloride desiccant solution

$51 \quad P C M \quad$ micro-encapsulated phase change material

$52 \quad P C M-\mathrm{LiCl}$ phase change enhanced $\mathrm{LiCl}$ desiccant solution

$53 \quad s \quad$ shell

54

55 1. Introduction

56 Air conditioning is essential to our lives and is greatly impacting the quality of our life and

57 even saves lives during intense heat waves [1]. The amount of installed air conditioning systems

58 is expected to increase dramatically in the coming decades, largely driven by economic growth

59 and global warming [2]. Nowadays, the majority of air conditioning systems used were

60 developed based on the vapour compression cycle [3]. These systems can control sensible load

61 effectively but are very inefficient to deal with latent load, in particular under hot and humid

62 climatic conditions. This is because a significant amount of energy is required to dehumidify

63 the air by overcooling the air below its dew point temperature in order to remove the moisture 
through condensation and then heating it to the desired temperature [4].

Liquid desiccant cooling as one of the alternative solutions has received increasing attention for air conditioning and dehumidification due to its effectiveness in humidity control and great potentials in energy savings [5-7]. A liquid desiccant is generally a concentrated salt solution such as lithium bromide $(\mathrm{LiBr})$, lithium chloride $(\mathrm{LiCl})$, and calcium chloride $\left(\mathrm{CaCl}_{2}\right)$ that directly absorbs moisture without cooling the air below its dew point. Liquid desiccant cooling can be driven by low-grade thermal energy $\left(50-90^{\circ} \mathrm{C}\right)$ such as waste heat or solar energy [8]. As the building peak cooling demand generally occurs at approximately the same time as local peak solar radiation, this opens up opportunities of using solar energy to drive liquid desiccant cooling and even to take air conditioning off the grid with the assistance of thermal energy storage systems.

Various types of liquid desiccant cooling systems and their potentials to maintain acceptable indoor thermal comfort under different climatic conditions have been studied. Ham et al. [9], for instance, developed a liquid desiccant and dew point evaporative cooling assisted $100 \%$ outdoor air system. The simulation results showed that $12 \%$ of the primary energy can be saved by using this system, in comparison with a typical variable air volume system. Elmer et al. [10] developed a liquid desiccant cooling system consisting of a regenerator, a dehumidifier and an evaporative inter-cooler integrated with an energy exchanger. The experimental results showed that the dehumidification effectiveness of this system was $30-47 \%$ with an average electrical COP of 2.5. Chen et al. [11] proposed a liquid desiccant dehumidifier and a regenerative indirect evaporative cooling system for fresh air treatment. The thermal 
energy obtained from solar collectors was used for liquid desiccant regeneration. The results showed that the energy saving of this system was $22.4-53.2 \%$ under various inlet air conditions, in comparison to a conventional chilled water air conditioning system. A solar driven liquid desiccant cooling system was developed by a company named L-DCS Technology [12]. This system was installed in a building in Singapore and used for cooling and dehumidification [13]. A dehumidifier is a key component in a liquid desiccant cooling system. Adiabatic dehumidifiers are relatively simple units, but they must work with a high desiccant flow rate and a high air flow rate in order to achieve a better dehumidification efficiency $[6,14]$. In adiabatic dehumidifiers, the temperature of the desiccant solution continuously increases along the desiccant flow direction. The increase in the solution temperature deteriorates the dehumidification efficiency as the ability of a desiccant solution to attract water vapour from an air stream decreases with the increase of the solution temperature and the decrease of the solution concentration [15]. In order to ensure dehumidifiers work with high efficiency and minimise the carryover of desiccant droplets, internally cooled dehumidifiers which can allow the desiccant solution working in a low temperature and low flow rate condition were studied [16]. The performance comparison of an adiabatic dehumidifier with an internally cooled dehumidifier showed that the dehumidification effectiveness of the internally cooled dehumidifier was improved from $0.3876-0.4771$ to $0.4769-0.7058$, in comparison to that of the adiabatic dehumidifier [17]. However, the inherent complex configuration of the internally cooled dehumidifiers and high maintenance costs make these systems less attractive.

$$
\text { Phase change materials (PCMs) with an ability to provide high energy storage densities and }
$$


106 the characteristics to store thermal energy at relatively constant temperatures have attracted

107 wide attention for developing high-performance buildings $[18,19]$. The development of PCMs

108 and PCM thermal energy storage for liquid desiccant cooling systems have been reported in a

109 few studies [20-22]. Niu et al. [20], for instance, proposed to use micro-nanoencapsulated

110 PCMs as a heat transfer fluid to improve the performance of internally cooled dehumidifiers in

111 liquid desiccant cooling systems. The micro-nanoencapsulated PCMs were prepared and their

112 thermo-physical properties were characterised. However, the real application of such materials

113 in dehumidifiers was not reported. Al-Abidi et al. [21] and Mahdi and Nsofor [22] respectively

114 developed a triplex tube PCM thermal energy storage for liquid desiccant cooling systems. Al-

115 Abidi et al. [21] experimentally tested the charging performance of the PCM thermal storage

116 unit using a paraffin wax with a melting temperature of $82{ }^{\circ} \mathrm{C}$ while Mahdi and Nsofor [22]

117 numerically investigated the charging performance of the thermal energy storage using the same

118 paraffin wax but was enhanced by alumina nanoparticles and a porous copper foam. However,

119 both studies did not integrate the PCM thermal energy storage into liquid desiccant cooling 120 systems.

121 In this study, a novel phase change enhanced LiCl (i.e. PCM-LiCl) desiccant solution was

122 proposed to improve the dehumidification efficiency of adiabatic dehumidifiers. The new

123 solution was prepared by dispersing micro-encapsulated PCMs (MPCMs) into LiCl desiccant

124 solutions. As MPCMs have a relatively large thermal storage capacity, the dispersion of

125 MPCMs into liquid desiccant solutions can ensure the mixture work under a low temperature

126 condition, improving overall dehumidification efficiency. The properties of the new PCM-LiCl 
127 desiccant solution such as density, enthalpy-temperature $(h-T)$ relationship, particle size

128 distribution, thermal conductivity, and vapour pressure were characterised through either

129 experimental measurements or theoretical investigation.

\section{Development and characterisation of PCM-LiCl desiccant solutions}

131 Dehumidifiers can generally offer a better performance when the desiccant solution is

132 working in a low temperature condition such as in the range of $20-30{ }^{\circ} \mathrm{C}$ [23-24]. As shown in

133 Fig. 1a [25], the temperature of the $\mathrm{LiCl}$ desiccant solution is continuously increased along the

134 height of the adiabatic dehumidifier due to the heat and mass transfer between the process air

135 and the liquid desiccant and the absorption heat released during the dehumidification process.

136 This increased temperature will lead to the increase in the vapour pressure of the liquid

137 desiccant and therefore decrease the vapour pressure difference between the process air and

138 liquid desiccant, which deteriorates the moisture transfer and dehumidification effectiveness of

139 the adiabatic dehumidifier. This deterioration can be further confirmed by the results (see Fig.

140 1b) reported in another study [26], in which it was shown that the mass flux of moisture between

141 the process air and liquid desiccant decreased along the flow direction of the liquid desiccant.

142 As PCMs have a large storage density, the dispersion of MPCMs into liquid desiccants can

143 improve the thermal capacity of the mixture and therefore can decrease the temperature increase

144 of the liquid desiccant along the flow direction of the adiabatic dehumidifier, improving overall

145 dehumidification effectiveness. 


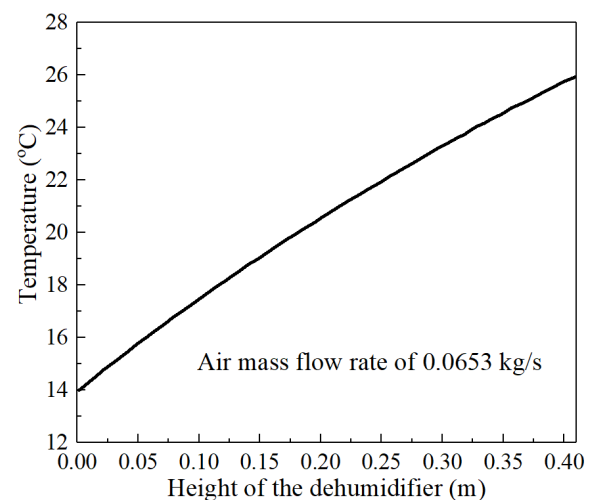

a) Temperature of the liquid desiccant [25]

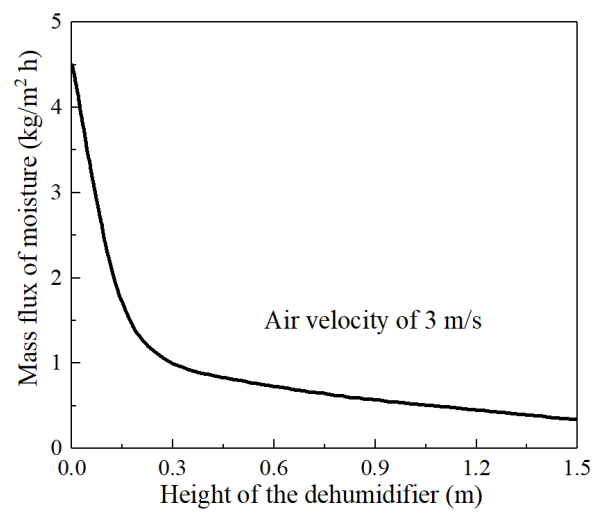

b) Mass flux of moisture [26]

Fig. 1. Temperature of $\mathrm{LiCl}$ liquid desiccant and mass flux of moisture along the flow direction of an adiabatic dehumidifier. and Micronal DS 5040X with a melting temperature of $25^{\circ} \mathrm{C}$ and $23{ }^{\circ} \mathrm{C}$ respectively, were used to prepare phase change enhanced $\mathrm{LiCl}$ desiccant solutions. Both MPCMs were made of a

155 highly crosslinked polymethylmethacrylate (PMMA) polymer wall and paraffin inside as the 156 PCM. It is noted that the MPCMs used in this study might not be optimal. As the temperature

157 of the inlet working solution of the dehumidifier is a controlled variable, the phase change

158 materials should be selected based on the set-point of the inlet working solution temperature in

159 order to ensure that the PCM undergoes the phase change process during the dehumidification. 
160 Deionized water and $\mathrm{LiCl}$ with a purity higher than $98 \%$ were used to prepare $\mathrm{LiCl}$ desiccant

161 solutions. The PCM-LiCl desiccant solution was prepared through directly dispersing the

162 MPCM particles into the $\mathrm{LiCl}$ desiccant solution via mechanical stirring.

\subsection{Characterisation of PCM-LiCl desiccant solutions}

164

To understand the likely benefits of using this new working solution for air

dehumidification, the properties of the PCM-LiCl desiccant solutions, including density, $h-T$

relationship, particle size distribution, thermal conductivity, and vapour pressure should be first characterised. In this study, these properties were characterised through either theoretical analysis or experimental measurements.

\subsubsection{Density}

The density of the PCM-LiCl desiccant solution was determined based on the density of each insoluble component of the mixture as the potential non-homogeneity of the new solution might introduce errors in measurements of the mixture. As the PMMA has good resistance to

$173 \mathrm{LiCl}$ and is insoluble in $\mathrm{LiCl}$ desiccant solutions, the density of the PCM-LiCl desiccant

174 solution can be calculated using Eq. (1) [27], in which the density of the MPCM was measured 175 using a pycnometer (to be introduced in Section 2.2) and the density of the LiCl desiccant

176 solution was determined using the correlation expressed in Eq. (2) [28].

179 where $\rho$ is the density, $\alpha_{i}$ are the parameters and the values of $\alpha_{0}-\alpha_{3}$ were 1.0, 0.540966 , - 
182 phase change material, and $\mathrm{LiCl}$ desiccant solution, respectively.

183

184

185

186

187

188

189

190

191

192

193

194

195

196

197

198

199

\subsubsection{Enthalpy-temperature $(h-T)$ relationship}

Differential scanning calorimeter (DSC) tests are commonly used to measure $h-T$ relationships of PCMs. In this study, the $h$ - $T$ relationship of the PCM-LiCl desiccant solution was not measured directly as $\mathrm{LiCl}$ desiccant solutions may corrode the metal container of the DSC device and further damage the equipment. Thus, the $h-T$ relationship of the new solution was determined using Eq. (3), based on the enthalpy of the $\mathrm{LiCl}$ desiccant solution determined using Eq. (4) [29] and the DSC test results of the MPCMs.

$h_{P C M-L i C l}=x_{m, P C M} h_{P C M}+\left(1-x_{m, P C M}\right) h_{L i C l}$

$h_{L i C l}=A+B T+C T^{2}$

where $h$ is the enthalpy, $T$ is the temperature, and the coefficients of $A, B$, and $C$ are calculated using Eqs. (5-7) [29], respectively.

$A=-66.2324+11.2711 x_{m, L i C l}-0.79853 x_{m, L i C l}^{2}+(2.1534 \mathrm{E}-02) x_{m, L i C l}^{3}-(1.66352 \mathrm{E}-04) x_{m, L i C l}^{4}$

$B=4.5751-0.146924 x_{m, L i C l}+(6.307226 \mathrm{E}-03) x_{m, L i C l}^{2}-$

$(1.38054 \mathrm{E}-04) x_{m, \mathrm{LiCl}}^{3}+(1.06690 \mathrm{E}-06) x_{m, \mathrm{LiCl}}^{4}$

$C=(-8.09689 \mathrm{E}-04)+(2.18145 \mathrm{E}-04) x_{m, L i C l}-(1.36194 \mathrm{E}-05) x_{m, L i C l}^{2}+(3.20998 \mathrm{E}-07) x_{m, \mathrm{LiCl}}^{3}-$

$(2.64266 \mathrm{E}-09) x_{m, L i C l}^{4}$

\subsubsection{Thermal conductivity}

The thermal conductivity of the PCM-LiCl desiccant solution was calculated using 
Maxwell's equation as shown in Eq. (8), which has been widely used to calculate the thermal

203 conductivity of MPCM suspensions [27, 30]. The thermal conductivity of the MPCM particles

$204\left(k_{P C M}\right)$ was determined using the composite sphere approach reported by Goel et al. [30], in

205 which the heat transfer resistance of the shell material was determined based on the thickness

206 of the MPCM shell. The heat transfer resistance of the core material was evaluated based on

207 the assumption that a solid sphere is in an infinite medium [31]. The thermal conductivity of

208 the MPCM can then be calculated using Eq. (9) [30]. The diameter of the MPCM particle was

209 measured using a particle size analyser to be introduced in Section 2.2 and the thickness of the

210 PMMA shell was determined using the composition of the MPCM measured using a

211 thermogravimetric analyser (see Section 2.2). The thermal conductivity of the $\mathrm{LiCl}$ desiccant

212 solution is calculated using Eq. (10) [28].

$213 \frac{k_{P C M-L i C l}}{k_{L i C l}}=\frac{2 k_{L i C l}+k_{P C M}+2 x_{v, P C M}\left(k_{P C M}-k_{L i C l}\right)}{2 k_{L i C l}+k_{P C M}-x_{v, P C M}\left(k_{P C M}-k_{L i C l}\right)}$

$214 \frac{1}{k_{P C M} d_{P C M}}=\frac{1}{k_{c} d_{c}}+\frac{d_{P C M^{-}}-d_{c}}{k_{s} d_{P C M} d_{c}}$

$215 k_{L i C l}=k_{H 2 O}-\alpha_{R} x_{m, L i C l, e}$

216 where $k$ is the thermal conductivity, $x_{v}$ is the volume fraction, $d$ is the diameter, $\alpha_{R}$ is the

217 equivalent thermal conductivity depression, $x_{m, L i C l, e}$ is the equivalent ionic concentration, and

218 the subscripts $s$ and $c$ indicate shell and core, respectively.

219 2.1.4 Vapour pressure

220 The dehumidification performance of a liquid desiccant is directly influenced by the vapour

221 pressure difference between the liquid desiccant and process air [5]. In this study, the vapour

222 pressure of the PCM-LiCl desiccant solution was measured using a thermogravimetric method 
223 [32]. In this method, the mass loss of the sample is measured using a thermogravimetric 224 analyser (i.e. TGA 5500 from TA Instruments was used in this study) and the result is then used 225 to determine the vapour pressure of the solution by using the Langmuir equation for free 226 vaporisation, as shown in Eq. (11) [33].

$\log \left(P_{T}\right)=\beta_{1} \log \left(\frac{1}{\beta_{2}}\left|\frac{d m}{d t}\right|_{T}\right)+\beta_{3}$

228 where $\left|\frac{d m}{d t}\right|_{T}$ and $P_{T}$ are the mass loss rate and the vapour pressure at a given temperature 229 respectively, and $\beta_{1}, \beta_{2}$ and $\beta_{3}$ are the coefficients influenced by the geometry structure of the 230 crucible. In this study, these three coefficients were determined based on the experimental 231 results for a reference substance (i.e. pure water) with the known vapour pressure. on the lid, as illustrated in Fig. 2a. The crucible was then placed on the holder of the balance 234 inside the thermogravimetric analyser (Fig. 2b). The sample was purged by a pure nitrogen gas 235 flow in order to maintain the water content outside the crucible close to zero.

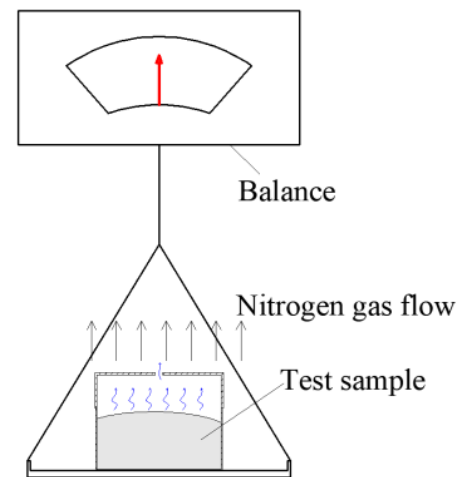

a) Schematic of vapour pressure measurement 


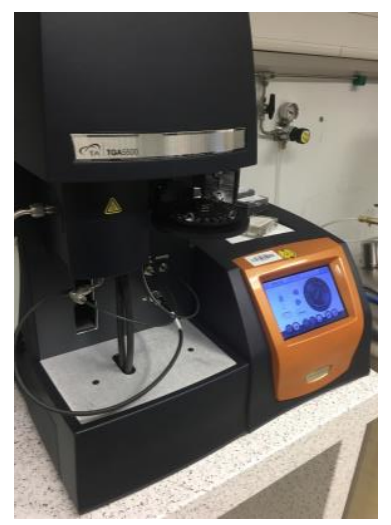

b) Thermogravimetric analyser used in this study

Fig. 2. Vapour pressure measurement using a thermogravimetric analyser.

\subsection{Characterisation of MPCMs and pure $\mathrm{LiCl}$ desiccant solutions}

The properties of the MPCMs were determined either using the information provided in

244 the product data sheet [34] or through the experimental measurements. provided in the datasheet [34]. However, the particle density of the MPCM was required to determine the density of the PCM-LiCl desiccant solutions. In this study, a pycnometer was used to measure the particle density of the MPCM. tests were implemented using a micro DSC (micro DSC III, SETARAM) device with a weight of the samples of around $300 \mathrm{mg}$. The heating/cooling rate used was $0.05 \mathrm{~K} / \mathrm{min}$. size analyser and the sample was pre-processed in an ultrasonic bath for deagglomeration of the particles before the measurement. The results were analyzed using Mie model [35], which worked well for homogeneous and spherical particles with a diameter less than $30 \mu \mathrm{m}[36,37]$.

256 The Mie model was derived by solving Maxwell's equations describing electromagnetic 
radiation for the light scattered by a homogeneous sphere under the uniform illumination [35]. Thermogravimetric analysis was carried out using a thermogravimetric analyser to determine the composition of the MPCMs, and then to calculate the thickness of the PMMA shell. During the test, the MPCM was heated to a temperature of $600{ }^{\circ} \mathrm{C}$ with a scanning rate of $1.0 \mathrm{~K} / \mathrm{min}$, and the test was then carried out using a nitrogen gas flow.

$\mathrm{As} \mathrm{LiCl}$ is highly hygroscopic and pure $\mathrm{LiCl}$ may absorb the moisture from the air during the preparation. A thermogravimetric analysis test for $\mathrm{LiCl}$ desiccant solutions was therefore carried out to determine the mass fraction of $\mathrm{LiCl}$ in the solution. During the measurement, the $\mathrm{LiCl}$ desiccant solution was heated to a temperature of $350{ }^{\circ} \mathrm{C}$ with a scanning rate of $5.0 \mathrm{~K} / \mathrm{min}$ and was then maintained at $350{ }^{\circ} \mathrm{C}$ for 18 hours [38]. The thermogravimetric analysis test was also performed with a nitrogen gas flow.

\section{Results of properties characterisation}

In this section, the properties of the MPCMs and PCM-LiCl desiccant solutions were respectively characterised using the methods introduced in Section 2. The majority of the tests presented in this study were repeated in order to confirm the consistency of the results.

\subsection{Results of characterisation of MPCM properties}

The measured densities of the MPCM DS 5038X and MPCM DS 5040X were 990.9 \pm 0.6 $\mathrm{kg} / \mathrm{m}^{3}$ and $1012.6 \pm 0.4 \mathrm{~kg} / \mathrm{m}^{3}$, respectively. These results were considered to be reasonable as the density of the two major compositions, i.e. paraffin wax and PMMA, were around $850 \mathrm{~kg}$ $\mathrm{m}^{-3}$ and $1150 \mathrm{~kg} \mathrm{~m}^{-3}$, respectively.

The DSC curves of the MPCM DS 5038X and MPCM DS 5040X are presented in Fig. 3 
278 and their onset temperatures, peak temperatures, and heat of fusion during the cooling and

279 heating processes are summarized in Table 1. The heat of fusion of the MPCM was calculated 280 based on the temperature range of $15-28{ }^{\circ} \mathrm{C}$. It can be observed that the peak phase change 281 temperatures of the MPCM DS 5038X for both heating and cooling were slightly higher than 282 those of the MPCM DS 5040X. The results from Table 1 also showed that the onset 283 temperatures of the MPCM DS 5038X were higher than those of the MPCM DS 5040X during 284 both cooling and heating processes.

285 Table 1. Onset temperatures, peak temperatures, and heat of fusion of the two MPCMs.

\begin{tabular}{lllllll}
\hline & DS 5038X & \multicolumn{5}{c}{ DS 5040X } \\
\hline & Onset & Peak & Heat of & Onset & Peak Temp. & $\begin{array}{l}\text { Heat of } \\
\text { fusion }(\mathrm{J} / \mathrm{g})\end{array}$ \\
& Temp. $\left({ }^{\circ} \mathrm{C}\right)$ & Temp. $\left({ }^{\circ} \mathrm{C}\right)$ & fusion $(\mathrm{J} / \mathrm{g})$ & Temp. $\left({ }^{\circ} \mathrm{C}\right)$ & $\left({ }^{\circ} \mathrm{C}\right)$ & 94.8 \\
Heating & 22.55 & 25.37 & 96.7 & 19.87 & 24.64 & 93.2 \\
\hline
\end{tabular}

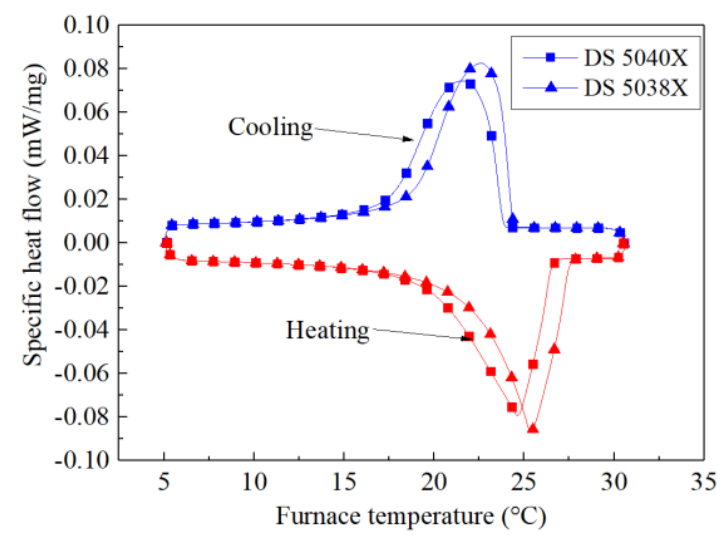

Fig. 3. DSC test results of DS 5038X and DS 5040X. 


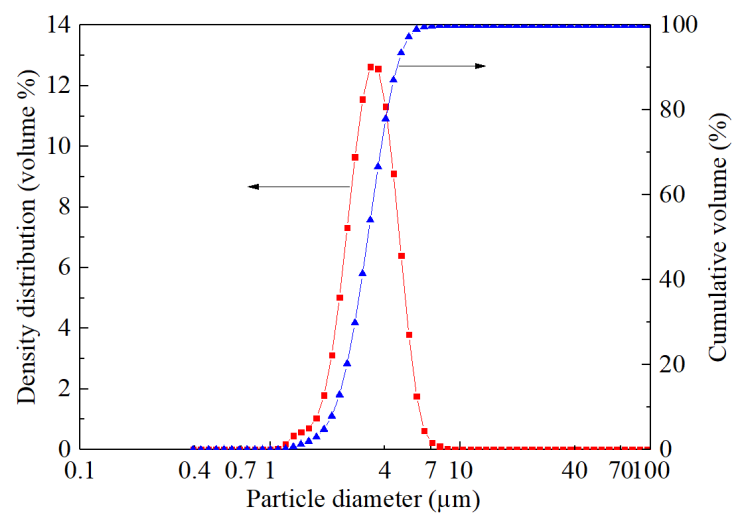

a) DS 5038X

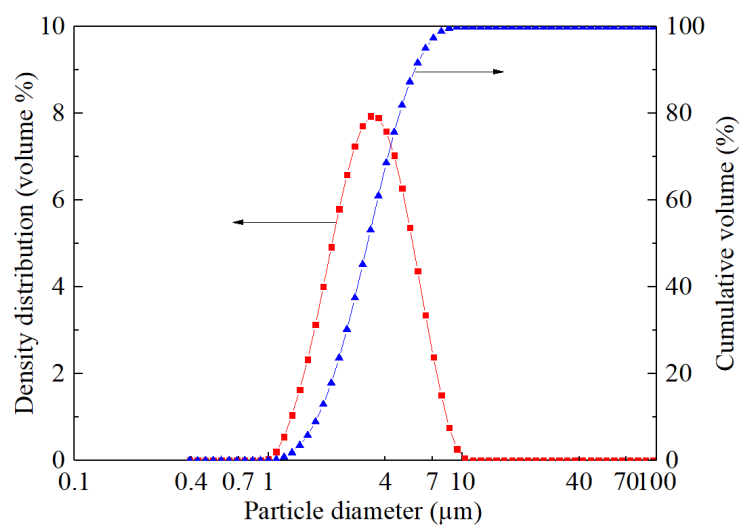

b) DS 5040X

Fig. 4. Particle size distribution of the two MPCMs.

The results (i.e. percentage weight and mass loss rate of the sample) from the

299 thermogravimetric analysis of the MPCMs DS 5038X and DS 5040X and a sample of the

300 MPCM DS 5038X after the decomposition test are presented in Fig. 5. The thermogravimetric

301 analysis results of the shell material (i.e. PMMA) obtained from [39] were also provided. It can

302 be seen that the thermal degradation of the MPCM DS 5038X can be divided into three phases

303 and the MPCM was mainly decomposed in the first two phases at a temperature below $330{ }^{\circ} \mathrm{C}$,

304 and the PMMA was completely decomposed at a temperature of around $454{ }^{\circ} \mathrm{C}$ (Fig. 5a).

305 Similar trends were also observed for the MPCM DS 5040X (Fig. 5b). By comparing the 306 thermogravimetric analysis curve of the MPCM to that of the PMMA, it can be derived that the 


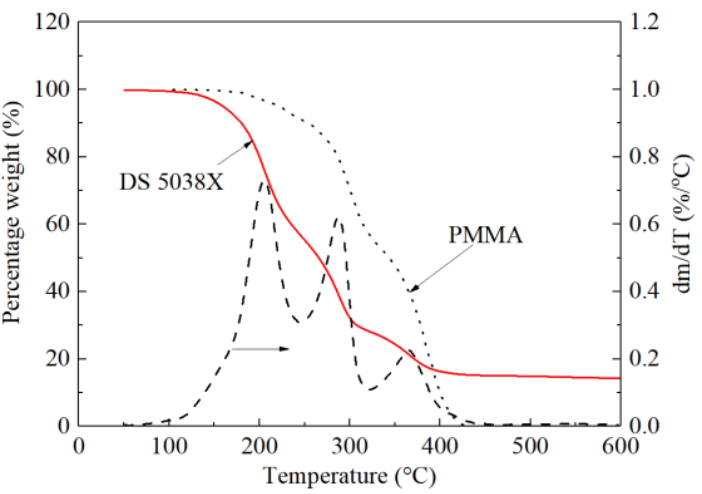

a) DS 5038X

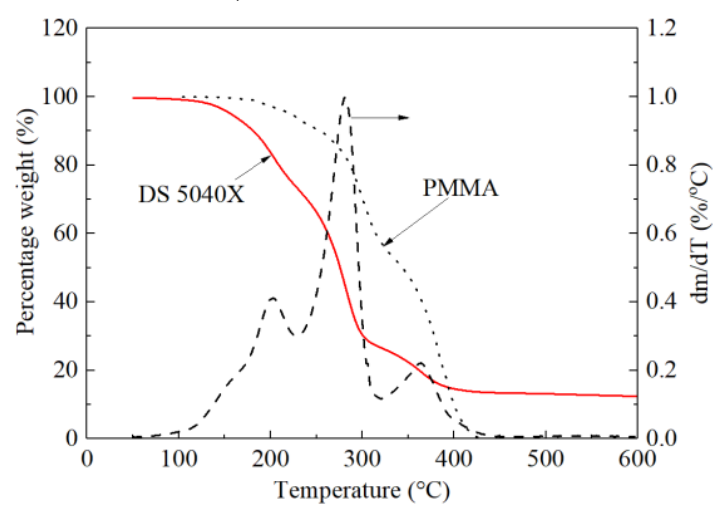

b) DS 5040X

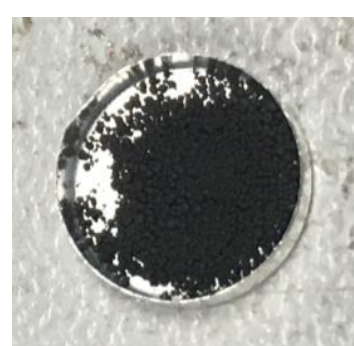

c) DS $5038 \mathrm{X}$ after the decomposition test Fig. 5. Decomposition of PMMA [39], MPCM DS 5038X and MPCM DS 5040X.

\subsection{Results of characterisation of PCM-LiCl desiccant solutions}

3.2.1 Verification of vapour pressure measurement

The vapour pressure of the $\mathrm{LiCl}$ desiccant solution was measured using the 
321 thermogravimetric method and the results were compared with the calculated values determined

322 using the correlation expressed in Eq. (12) [28]. The concentration of the LiCl desiccant

323 solution was first determined via a thermogravimetric analysis test and the vapour pressure of

324 the $\mathrm{LiCl}$ desiccant solution was then measured at a temperature of $50{ }^{\circ} \mathrm{C}$ using the method

325 presented in Fig. 2. The test results are presented in Fig. 6, which were measured based on the

326 initial concentration of the $\mathrm{LiCl}$ solution of $29.53 \%$. It can be found that the vapour pressure of

327 the solution decreased with the increase of the solution concentration and the measured values

328 generally agreed well with the calculated results. The average and maximum deviations

329 between the measured and calculated values were $1.9 \%$ and 5.5\%, respectively. It is worthwhile

330 to note that the total mass of the test sample used was relatively small due to the capacity of the

331 crucible used and the increase of the concentration was resulted by the continuous loss of the

332 water in the solution.

$333 P_{L i C l}=\pi_{25} f\left(x_{m, L i C l}, \theta\right) P_{H 2 O}$

334 where $\theta$ is the reduced temperature, $f\left(x_{m, L i C l}, \theta\right)$ is determined using Eq. (13) [28], and $\pi_{25}$ is

335 calculated using Eq. (14) [28].

336

$f\left(x_{m, L i C l}, \theta\right)=2-\left[1+\left(\frac{x_{m, L i C l}}{\pi_{0}}\right)^{\pi_{1}}\right]^{\pi_{2}}+\left\{\left[1+\left(\frac{x_{m, L i C l}}{\pi_{3}}\right)^{\pi_{4}}\right]^{\pi_{5}}-1\right\} \theta$

337

$\pi_{25}=1-\left[1+\left(\frac{x_{m, L i C l}}{\pi_{6}}\right)^{\pi_{7}}\right]^{\pi_{8}}-\pi_{9} \exp \left(-\frac{\left(x_{m, L i C l}-0.1\right)^{2}}{0.005}\right)$

338 where $\pi_{0-} \pi_{8}$ are the coefficients and the values used were provided in Table 2 [28]. 


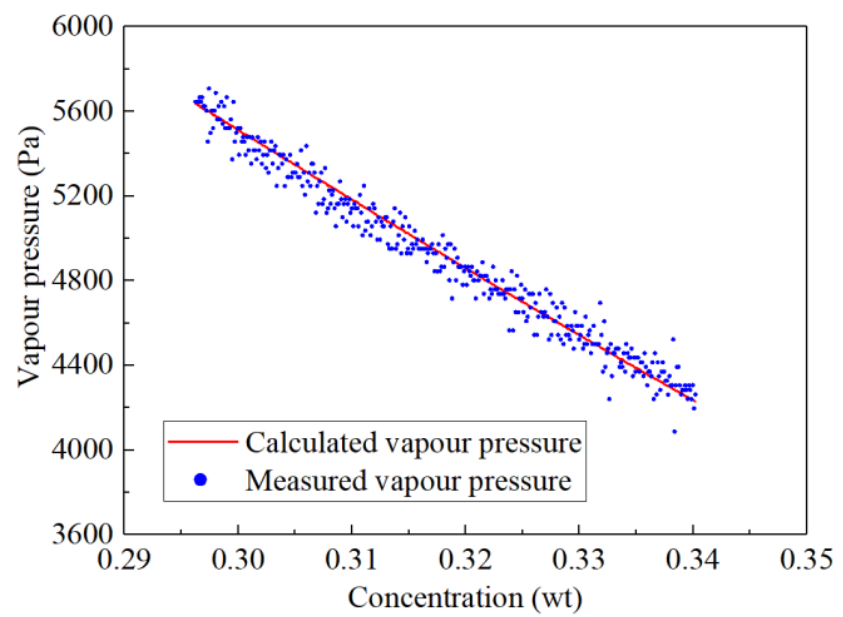

Fig. 6. Measured and calculated vapour pressures of the $\mathrm{LiCl}$ desiccant solution.

342 Table 2. Coefficients for vapour pressure calculation of $\mathrm{LiCl}$ desiccant solutions [28].

\begin{tabular}{llllllllll}
\hline$\pi_{0}$ & $\pi_{1}$ & $\pi_{2}$ & $\pi_{3}$ & $\pi_{4}$ & $\pi_{5}$ & $\pi_{6}$ & $\pi_{7}$ & $\pi_{8}$ & $\pi_{9}$ \\
\hline 0.28 & 4.30 & 0.60 & 0.21 & 5.10 & 0.49 & 0.362 & -4.75 & -0.40 & 0.03 \\
\hline
\end{tabular}

\subsubsection{Properties characterisation}

In this study, the $\mathrm{LiCl}$ desiccant solution with a concentration of $35 \%$ was first prepared and the MPCMs with different mass fractions were then mixed with the $\mathrm{LiCl}$ desiccant solution to prepare the PCM-LiCl desiccant solutions. The densities of different PCM-LiCl desiccant

348 solutions were then calculated using Eqs. (1) and (2) and the results are presented in Fig. 7. It

349 can be seen that the density of the PCM-LiCl desiccant solutions decreased with the increase

350 of the mass fraction of the MPCMs as the density of the MPCMs was lower than that of the

351 LiCl desiccant solution. The density of the mixture using the MPCM DS 5038X was always

352 lower than that using the MPCM DS 5040X which was resulted by the relatively low density 
354 is applicable to homogenous fluids and the PCM-LiCl desiccant solution developed in this study

355 can be considered as homogenous when it is well mixed.

356 occurred in the MPCM melting temperature range of $20-27^{\circ} \mathrm{C}$. The enthalpy of the solution and (4). The heating curve of the MPCM obtained from the DSC measurement was used to evaluate the enthalpy of the MPCM. The results are presented in Fig. 8. The enthalpy of the $\mathrm{LiCl}$ desiccant solution without MPCMs was also presented in this figure. It can be observed that the enthalpy of the PCM-LiCl desiccant solution increased with the increase of the solution temperature and the mass fraction of the MPCMs in the mixture. A large increasing rate then increased almost linearly if further increasing the solution temperature. There was not a clear difference between the use of two different MPCMs.

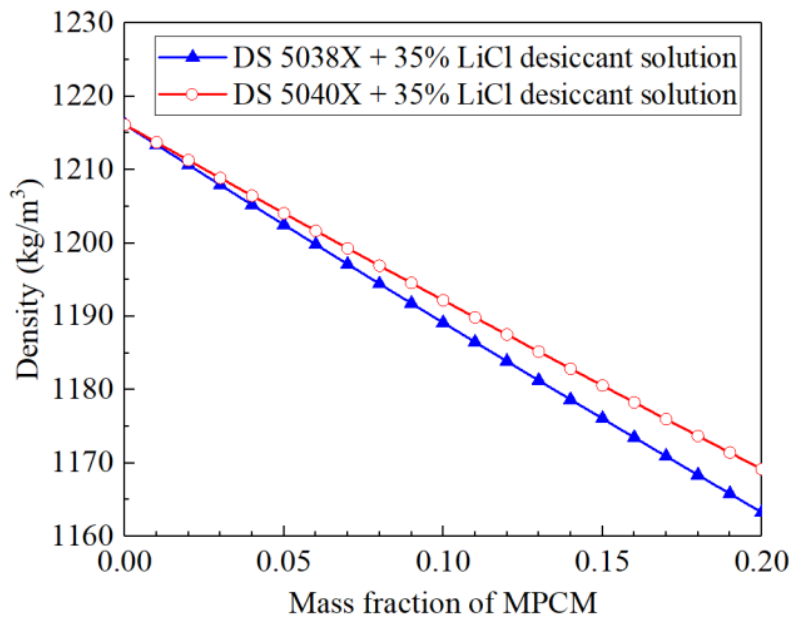

Fig. 7. Density of the PCM-LiCl desiccant solution. 


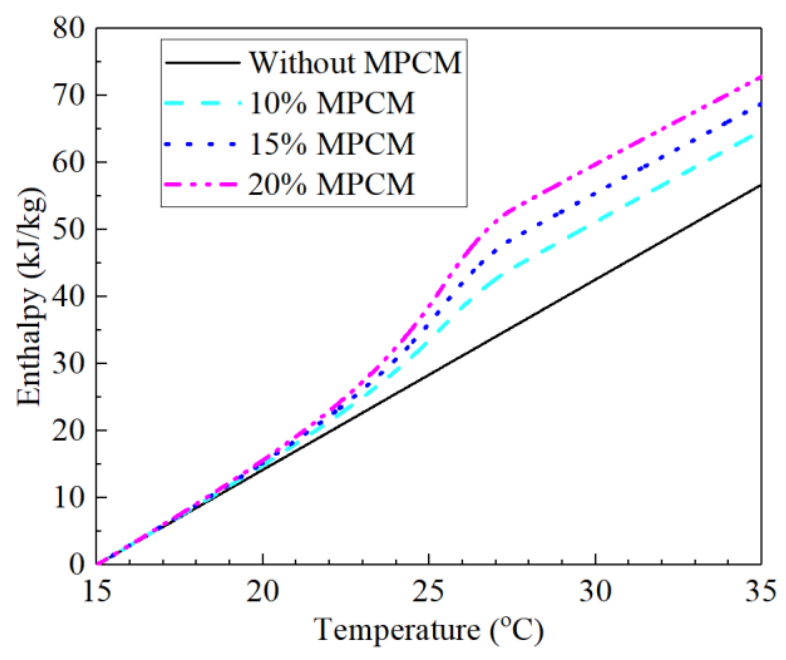

a) DS 5038X

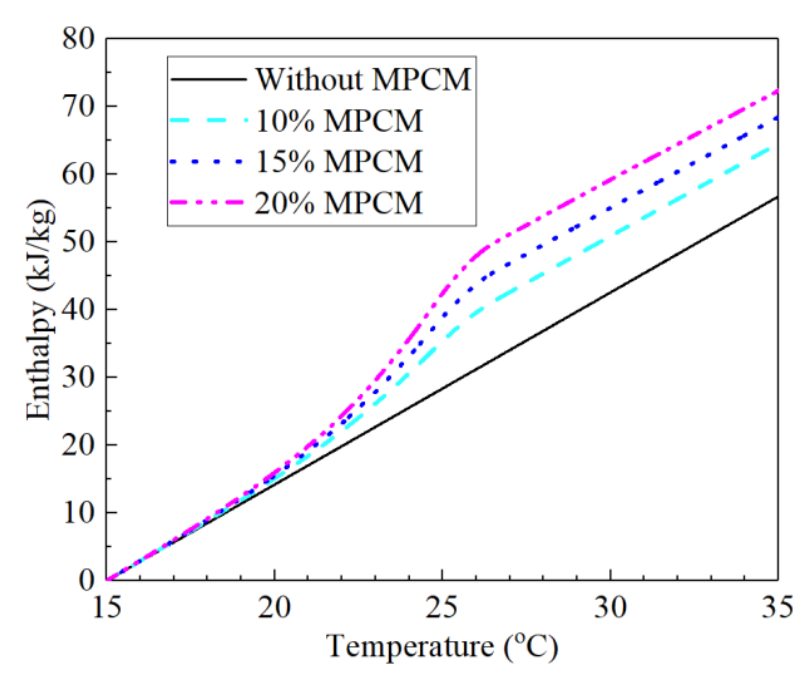

b) DS 5040X

371 Fig. 8. Enthalpy-temperature relationships of the pure $\mathrm{LiCl}$ desiccant solution and $\mathrm{PCM}-\mathrm{LiCl}$ desiccant solutions with different mass fractions of the MPCMs.

374 and (9) based on the composition and particle size of the MPCM determined. It is noted that

375 the diameter of the particles dispersed into the $\mathrm{LiCl}$ desiccant solution was assumed to be the

376 same as the volumetric average diameter of the particles. It was also assumed that the residual

377 of the MPCMs obtained from the thermogravimetric analysis had the same thermal conductivity 
as the PCM used. The resulted thermal conductivity of the PCM-LiCl desiccant solutions is

379 presented in Fig. 9. It can be observed that the thermal conductivities of the PCM-LiCl desiccant

380 solutions using the MPCM DS 5040X and DS 5038X were very close to each other when the

381 mass fraction of the MPCMs was less than 20\%. The thermal conductivity of the PCM-LiCl

382 desiccant solutions decreased with the increase of the mass fraction of the MPCMs as the

383 thermal conductivity of the MPCM particle was lower than that of the LiCl desiccant solution.

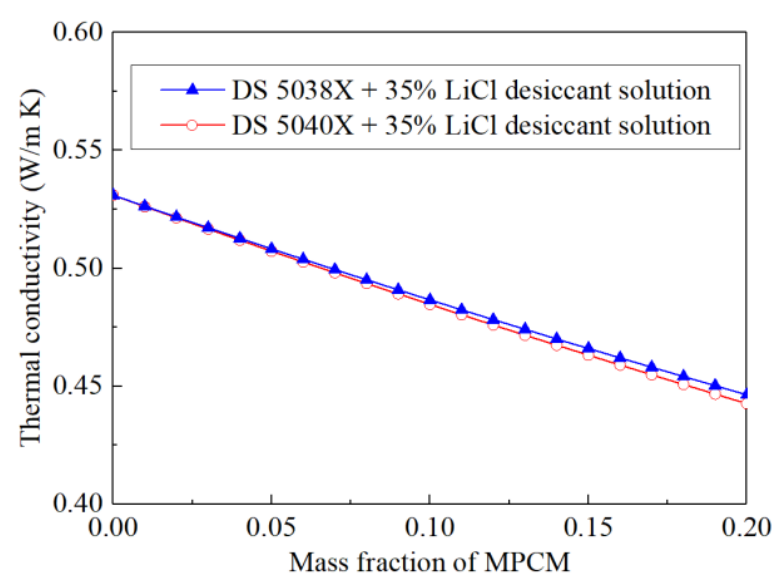

Fig. 9. Thermal conductivity of the PCM-LiCl desiccant solutions with different mass fractions of MPCMs.

The vapour pressures of the PCM-LiCl desiccant solutions with different mass fractions of the two MPCMs were measured using the thermogravimetric method. As the variation in the vapour pressures of the PCM-LiCl solutions using the two MPCMs showed a similar trend, the results of the PCM-LiCl solution using the MPCM DS 5040X under the temperature of $50{ }^{\circ} \mathrm{C}$ were presented only.

During the measurement, the $\mathrm{LiCl}$ desiccant solution was first prepared and the concentration of the $\mathrm{LiCl}$ desiccant solution was determined via a thermogravimetric analysis test. A relatively low initial concentration of the $\mathrm{LiCl}$ desiccant solution (i.e. 29.53\%) was used 
to prepare $\mathrm{PCM}-\mathrm{LiCl}$ desiccant solutions as the concentration of the desiccant solution was expected to increase during the vapour pressure measurement due to continuous loss of the water in the solution. The PCM-LiCl desiccant solution was then prepared in the crucible presented in Fig. 2a. Table 3 summarises the details of the three samples used in the test. It is worthwhile to note that the mass fraction of the MPCM in each test sample cannot be precisely controlled during the preparation.

Table 3. Test samples prepared for vapour pressure measurement.

\begin{tabular}{llll}
\hline Test sample & Weight $(\mathrm{mg})$ & Mass fraction of the MPCM & Mass fraction of the LiCl \\
\hline Sample 1 & 49.158 & 0.029 & 0.288 \\
Sample 2 & 57.424 & 0.128 & 0.258 \\
Sample 3 & 54.190 & 0.180 & 0.243 \\
\hline
\end{tabular}

403 under the temperature of $50{ }^{\circ} \mathrm{C}$ are presented in Figs. 10 and 11. The vapour pressures of $\mathrm{LiCl}$ desiccant solutions without the MPCM were also presented, which were calculated based on the same temperature condition and the same initial mass fractions of $\mathrm{LiCl}$ in the desiccant 408 solutions as those presented in Table 3. The vapour pressure of the pure $\mathrm{LiCl}$ desiccant solution 409 was determined using Eqs. (12)-(14). It can be seen that the vapour pressures of the PCM-LiCl 410 desiccant solutions of three samples continuously decreased as a function of time. This is 411 because the mass fractions of both MPCM and $\mathrm{LiCl}$ continuously increased with time during 412 the test due to the water loss (Fig. 11). For each test sample, the vapour pressure of the PCM$413 \mathrm{LiCl}$ desiccant solution was always lower than that of the pure $\mathrm{LiCl}$ desiccant solution during 
414 the test period due to the existence of the MPCM particles. The vapour pressure of the PCM-

$415 \mathrm{LiCl}$ desiccant solution decreased with the increase of the mass fraction of the MPCM in the

416 mixture.

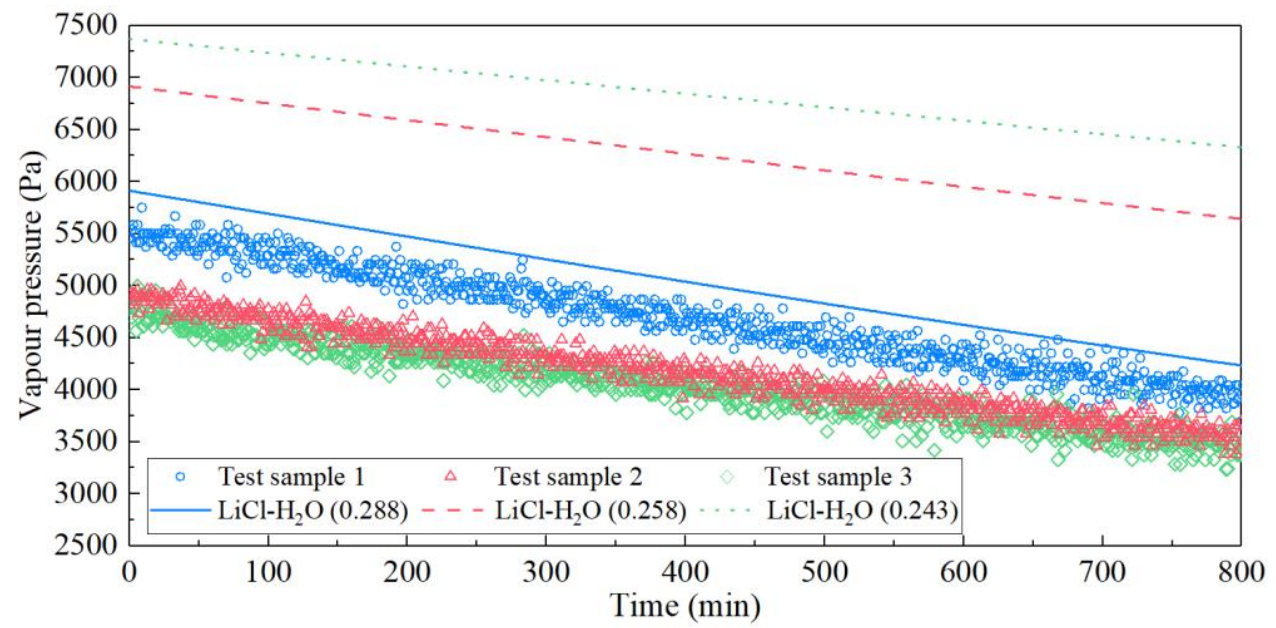

418 Fig. 10. Variation of vapour pressure of the PCM-LiCl desiccant solutions and pure $\mathrm{LiCl}$ desiccant solutions as a function of time.

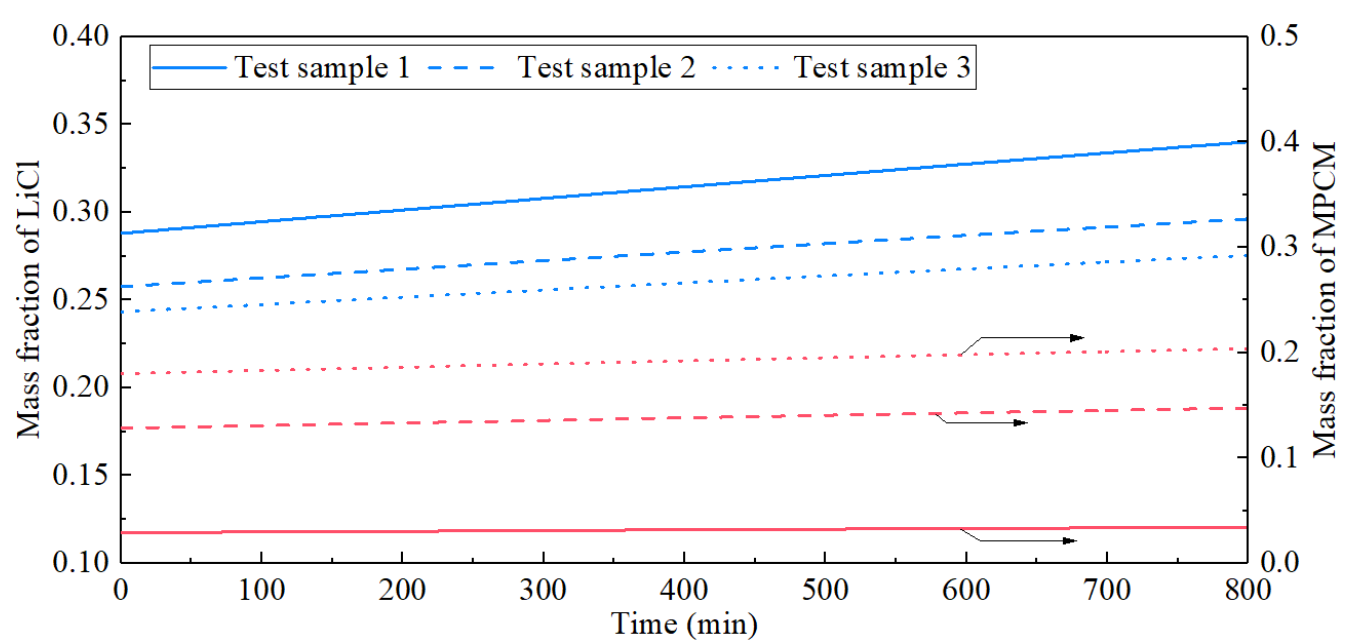

421 Fig. 11. Variation of mass fractions of the MPCM and $\mathrm{LiCl}$ in the PCM-LiCl desiccant solutions as a function of time. 
425

426

427

428

430

431

432

desiccant solution can decrease the vapour pressure and increase the thermal capacity of the mixture, which can improve the dehumidification efficiency of adiabatic dehumidifiers although the thermal conductivity of the mixture was slightly decreased. In principle, a low solution flow rate can be used in the liquid desiccant cooling system when using the PCM-LiCl desiccant solution due to the increased thermal capacity and reduced vapour pressure. It is also expected that the size of the dehumidifier using the new working solution can be decreased without compromising the dehumidification performance, when compared to the dehumidifier using pure desiccant solutions. In addition, the temperature of the inlet solution to the dehumidifier should be optimised in order to maximise the benefits of using MPCMs in the desiccant solution. It is also noteworthy that thermal regeneration might not be an optimal method for liquid desiccant cooling systems when using PCM-LiCl desiccant solutions and non-thermal regeneration methods may be required, which will be investigated in future studies. However, the potential benefits of using PCM-LiCl desiccant solutions should be evaluated in liquid desiccant cooling systems by considering the influence of the MPCM particles in both dehumidifiers and regenerators. Adding the MPCM particles into liquid desiccant solutions may increase the power consumptions of the circulation pump. Meanwhile, the mass fraction of the MPCMs in the mixture should be optimised. The long-term performance and potential segregation of MPCM particles in the new working solution should also be examined.

\section{Conclusions}

This study presented the development and characterisation of a new phase change enhanced working solution for liquid desiccant cooling systems. The new solution was prepared through 
447 fluid of the $\mathrm{LiCl}$ desiccant solution. The properties including density, $h-T$ relationship, thermal 448 conductivity, particle size distribution, and vapour pressure of the new solution were 449 characterised either through direct measurement or theoretical analysis. desiccant solution decreased with the increase of the mass fraction of the MPCMs in the mixture due to the lower density of the MPCMs used in comparison with the pure LiCl solutions. The thermal capacity of the PCM-LiCl desiccant solutions was substantially increased in the melting range of the MPCMs. The thermal conductivities of the PCM-LiCl desiccant solutions using the MPCMs DS 5038X and DS 5040X were very close to each other when the mass fraction of the MPCMs was less than $20 \%$ and the thermal conductivity decreased with the increase of the mass fraction of the MPCMs. The vapour pressure of the new working solution decreased due

458 to the existence of the MPCM particle as compared to the $\mathrm{LiCl}$ solution without using the

459 MPCMs. The dehumidification performance of adiabatic dehumidifiers could be potentially 460 improved by using this new working solution due to its decreased vapour pressure and increased 461 thermal capacity, which will be investigated in our future studies.

\section{Acknowledgement}

463 The first author would like to thank German Academic Exchange Service (DAAD) for 464 providing financial support to visit Fraunhofer Institute for Solar Energy Systems, Freiburg, 465 Germany, to carry out this work.

\section{References}


Association of weekly suicide rates with temperature anomalies in two different climate types,

[2] H. Chappells, E. Shove, Debating the future of comfort: environmental sustainability,

[3] R.C. Arora, Refrigeration and air conditioning, PHI Learning Pvt. Ltd., New Delhi, 2012.

[4] American Society of Heating, Refrigerating and Air-Conditioning Engineers (ASHRAE),

ASHRAE Handbook-Fundamentals, ASHRAE, Atlanta, 2009.

[5] L. Mei, Y.J. Dai, A technical review on use of liquid-desiccant dehumidification for airconditioning application, Renewable and Sustainable Energy Reviews 12 (3) (2008) 662-689.

[6] A.H. Abdel-Salam, C.J Simonson, State-of-the-art in liquid desiccant air conditioning

[7] Y. Guo, Z. Ma, A. Al-Jubainawi, P. Cooper, L.D. Nghiem, Using electrodialysis for

481 regeneration of aqueous lithium chloride solution in liquid desiccant air conditioning systems,

482 Energy and Buildings 116 (2016) 285-295.

483 [8] D.L. Shukla, K.V. Modi, A technical review on regeneration of liquid desiccant using solar 484 energy, Renewable and Sustainable Energy Reviews 78 (2017) 517-529.

485 [9] S.W. Ham, S.J. Lee, J.W. Jeong, Operating energy savings in a liquid desiccant and dew 486 point evaporative cooling-assisted 100\% outdoor air system, Energy and Buildings 116 (2016) 
488

489

490

491

492

493

494

495

496

497

498

499

500

501

502

503

504

505

506

507

508

[10]T. Elmer, M. Worall, S. Wu, S. Riffat, An experimental study of a novel integrated desiccant air conditioning system for building applications, Energy and Buildings 111 (2016) 434-445.

[11] Y. Chen, H. Yang, Y. Luo, Investigation on solar assisted liquid desiccant dehumidifier and evaporative cooling system for fresh air treatment, Energy 143 (2018) 114-127.

[12]L-DCS Technology, http://www.1-dcs.de/, 2018 (accessed 15 December 2018)

[13]A. Hublitz, Efficient Energy Storage in Liquid Desiccant Cooling Systems, Ph.D Thesis, Technische Universität München, München, Germany, 2008.

[14]M.M. Rafique, P. Gandhidasan, H.M. Bahaidarah, Liquid desiccant materials and dehumidifiers-A review, Renewable and Sustainable Energy Reviews 56 (2016) 179-195.

[15]N. Fumo, D.Y. Goswami, Study of an aqueous lithium chloride desiccant system: air dehumidification and desiccant regeneration, Solar Energy 72 (4) (2002) 351-361.

[16]A. Lowenstein, Review of liquid desiccant technology for HVAC applications, HVAC\&R Research 14 (6) (2008) 819-839.

[17]P. Bansal, S. Jain, C. Moon, Performance comparison of an adiabatic and an internally cooled structured packed-bed dehumidifier, Applied Thermal Engineering 31 (1) (2011) 14-19. [18]Z. Ma, W. Lin, M.I. Sohel, Nano-enhanced phase change materials for improved building performance, Renewable and Sustainable Energy Reviews 58 (2016) 1256-1268.

[19]Z. Hu, A. Li, R. Gao, H. Yin, A comparison study on melting inside the rectangular and curved unit with a vertical heating wall, Journal of Thermal Analysis and Calorimetry 122 (2) (2015) 831-842.

[20]X. Niu, Q. Xu, Y. Zhang, Y. Zhang, Y. Yan, T. Liu, Fabrication and properties of micro- 
nanoencapsulated phase change materials for internally-cooled liquid desiccant

510 dehumidification, Nanomaterials 7 (5) (2017) 96.

511 [21]A.A. Al-Abidi, S. Mat, K. Sopian, M.Y. Sulaiman, A. T. Mohammad, Experimental study 512 of PCM melting in triplex tube thermal energy storage for liquid desiccant air conditioning 513 system, Energy and Buildings 60 (2013) 270-279.

514 [22]J.M. Mahdi, E.C. Nsofor, Melting enhancement in triplex-tube latent heat energy storage 515 system using nanoparticles-metal foam combination, Applied Energy 191 (2017) 22-34.

516 [23]L. Wang, F. Xiao, X. Zhang, R. Kumar, An experimental study on the dehumidification 517 performance of a counter flow liquid desiccant dehumidifier, International Journal of 518 Refrigeration 70 (2016) 289-301.

519 [24]X.H. Liu, Y. Jiang, K.Y. Qu, Heat and mass transfer model of cross flow liquid desiccant 520 air dehumidifier/regenerator, Energy Conversion and Management 48 (2) (2007) 546-554.

521 [25]I.P. Koronaki, R.I. Christodoulaki, V.D. Papaefthimiou, E.D. Rogdakis, Thermodynamic 522 analysis of a counter flow adiabatic dehumidifier with different liquid desiccant materials, 523 Applied Thermal Engineering 50 (1) (2013) 361-373.

524 [26]M.R. Islam, S.W.L. Alan, K.J. Chua, Studying the heat and mass transfer process of liquid 525 desiccant for dehumidification and cooling, Applied Energy 221 (2018) 334-347.

526 [27]B. Chen, X. Wang, R. Zeng, Y. Zhang, X. Wang, J. Niu, Y. Li, H. Di, An experimental study 527 of convective heat transfer with microencapsulated phase change material suspension: laminar 528 flow in a circular tube under constant heat flux, Experimental Thermal and Fluid Science 32 (8) $529 \quad$ (2008) 1638-1646. 
530 [28]M.R. Conde, Properties of aqueous solutions of lithium and calcium chlorides:

531 formulations for use in air conditioning equipment design, International Journal of Thermal

532 Sciences 43 (4) (2004) 367-382.

533 [29]S.K. Chaudhari, K.R Patil, Thermodynamic properties of aqueous solutions of lithium

534 chloride, Physics and Chemistry of Liquids 40 (3) (2002) 317-325.

535 [30]M. Goel, S.K. Roy, S. Sengupta, Laminar forced convection heat transfer in 536 microcapsulated phase change material suspensions, International Journal of Heat and Mass 537 Transfer 37 (4) (1994) 593-604.

538 [31]E.C. Guyer, Handbook of applied thermal design, CRC Press, Philadelphia, 1999.

539 [32]D.M. Price, Vapor pressure determination by thermogravimetry, Thermochimica Acta (367)

$540 \quad$ (2001) 253-262.

541 [33]S. Giani, Determination of vapor pressure and the enthalpy of vaporization by TGA,

542 METTLER TOLEDO Thermal Analysis UserCom 38 (2014) 15-18.

543 [34]Micronal Product Manual, https://www.microteklabs.com/micronal, 2018 (accessed 10

544 December 2018).

545 [35]D.L. Black, M.Q. McQuay, M.P. Bonin, Laser-based techniques for particle-size 546 measurement: a review of sizing methods and their industrial applications, Progress in Energy 547 and Combustion Science 22 (3) (1996) 267-306.

548 [36]G.B. de Boer, C. de Weerd, D. Thoenes, H.W. Goossens, Laser diffraction spectrometry: 549 Fraunhofer diffraction versus Mie scattering, Particle \& Particle Systems Characterization 4 (1550 4) (1987) 14-19. 
551 [37] J. Giro-Paloma, G. Oncins, C. Barreneche, M. Martínez, A.I. Fernández, L.F. Cabeza, 552 Physico-chemical and mechanical properties of microencapsulated phase change material, 553 Applied Energy 109 (2013) 441-448.

554 [38]P. Masset, Thermogravimetric study of the dehydration reaction of $\mathrm{LiCl} \cdot \mathrm{H} 2 \mathrm{O}$, Journal of 555 Thermal Analysis and Calorimetry 96 (2) (2009) 439-441.

556 [39]M.C. Costache, D. Wang, M.J. Heidecker, E. Manias, C.A. Wilkie, The thermal degradation 557 of poly (methyl methacrylate) nanocomposites with montmorillonite, layered double 558 hydroxides and carbon nanotubes, Polymers for Advanced Technologies 17 (4) (2006) 272-280. 559 [40]J. Giro-Paloma, C. Barreneche, M. Delgado, M. Martínez, A.I. Fernández, L.F. Cabeza, 560 Physicochemical and thermal study of a MPCM of PMMA shell and paraffin wax as a core, $561 \quad$ Energy Procedia 54 (2014) 347-354. 